\title{
Quantum limited noise figure operation of high gain erbium doped fiber amplifiers
}

Lumholt, Ole; Povlsen, Jørn Hedegaard; Schüsler, Kim; Bjarklev, Anders Overgaard; Dahl-Petersen, Svend; Rasmussen, Thomas; Rottwitt, Karsten

Published in:

Journal of Lightwave Technology

Link to article, DOI:

$10.1109 / 50.254094$

Publication date:

1993

Document Version

Publisher's PDF, also known as Version of record

Link back to DTU Orbit

Citation (APA):

Lumholt, O., Povlsen, J. H., Schüsler, K., Bjarklev, A. O., Dahl-Petersen, S., Rasmussen, T., \& Rottwitt, K. (1993). Quantum limited noise figure operation of high gain erbium doped fiber amplifiers. Journal of Lightwave Technology, 11(8), 1344-1352. https://doi.org/10.1109/50.254094

\section{General rights}

Copyright and moral rights for the publications made accessible in the public portal are retained by the authors and/or other copyright owners and it is a condition of accessing publications that users recognise and abide by the legal requirements associated with these rights.

- Users may download and print one copy of any publication from the public portal for the purpose of private study or research.

- You may not further distribute the material or use it for any profit-making activity or commercial gain

- You may freely distribute the URL identifying the publication in the public portal 


\title{
Quantum Limited Noise Figure Operation of High Gain Erbium Doped Fiber Amplifiers
}

\author{
Ole Lumholt, Jørn H. Povlsen, Kim Schüsler, Anders Bjarklev,
} Svend Dahl-Petersen, Thomas Rasmussen, and Karsten Rottwitt

\begin{abstract}
Performance improvements by using an isolator as an amplified spontaneous emission suppressing component within erbium-doped fibers are evaluated. Simultaneous high-gain and near-quantum-limited noise figures can be obtained by such a scheme. The noise figure improves for input signal powers below $-5 \mathrm{dBm}$ and an improvement of $2.0 \mathrm{db}$ with a simultaneous gain increase of $4.1 \mathrm{~dB}$ is measured relative to a gain optimized fiber. The optimum isolator location is evaluated for different pump and signal wavelengths in both an $\mathbf{A l} / \mathbf{E r}$-doped and a $\mathbf{G e} / \mathbf{E r}$ doped fiber, for pump and signal power variations and different pump configurations. In all cases the optimum isolator position lies within $10-37 \%$ of the total fiber length for small signal operation.
\end{abstract}

\section{INTRODUCTION}

$\mathbf{T}$ HE rapid development within the field of optimization of gain efficiencies of erbium-doped fiber amplifiers (EDFA's) has resulted in a tremendous increase to $11 \mathrm{~dB} / \mathrm{mW}$ [1], and a gain as high as $51 \mathrm{~dB}$ has recently been reported [2]. However, even for such highly optimized fibers, most of the pump photons are still lost to amplify the spontaneously radiated emission, yielding gain saturation and a noise figure that departs considerably from the quantum limit. The latter is due to the strong interdependence between the noise figure and the population inversion in the input fiber end, which is heavily diminished by the amount of backward-travelling amplified spontaneous emission (ASE). A low noise figure can be obtained either by shortening the fiber well below its optimum length [3] or by increasing the input signal power to the region where neither the ASE nor the signal saturation will be determinative upon the population inversion [4]. However, both options result in a considerable decrease in gain efficiency.

Essential demands for pre-amplifier or in-line amplifier operation of the EDFA are to achieve simultaneous high gain and a noise figure close to the quantum limit. An obvious way to comply with both requirements is to insert an isolator as an ASE attenuating component within the active fiber length [5]. Earlier reports on amplifier measurements have shown

Manuscript received August 5, 1992; revised February 19, 1993. This work was supported by the Danish Technical Research Council and by the National Agency of Industry and Trade, Denmark.

O. Lumholt, J. H. Povlsen, A. Bjarklev, T. Rasmussen, and K. Rottwitt are with the Center for Broadband Telecommunications, Electromagnetics Institute, Technical University of Denmark, DK-2800 Lyngby, Denmark.

K. Schüsler is with the NKT Research Center A/S, Sognevej 11, DK-2605 Brøndby, Denmark.

S. Dahl-Petersen is with NKT Elektronik, NKT Alle 85, DK-2605, Brøndby, Denmark. setups where an isolator is inserted between two EDFA's in succession, but for quite different intentions. In [6] two different pump wavelengths are used with the isolator in order to prevent gain saturation by the $0.98-\mu \mathrm{m}$ pump amplifying the 1.48- $\mu \mathrm{m}$ light. Another example is shown in [7] where gain equalization is performed by using a filter between fibers with different co-doping. An isolator is inserted in order to suppress reflections from the filter.

In this paper the aim is to analyze the amplifier performance improvements obtained by using the isolator as a population inversion enriching component. The optimum isolator location is evaluated and the improvements are analyzed for a germanium as well as an aluminum co-doped EDFA. The evaluation is performed for both $0.98 \mu \mathrm{m}$ and $1.47 \mu \mathrm{m}$ pumping, for different signal wavelengths, and for different pump and signal power levels and different pump configurations. Finally, an experimental verification is presented.

\section{BACKGROUND FOR USE OF ISOLATOR}

All simulations presented in this paper are evaluated by use of an accurate numerical model that is presented in [8], [5]. Input to the model are the fiber design and the measured crosssection spectra of emission and absorption [9], as shown in Fig. 1. A high NA Al-co-doped and a high NA Ge-co-doped erbium fiber with index profiles (shown as inserts in Fig. 1) and with Er profiles following the index distribution are considered. The central dip in the profile of the Ge-doped EDFA, which is due to vaporization of dopants during collapse of the preform, has no significant influence on the performance of EDFA's with high NA.

In order to describe the effects of including an isolator within an EDFA, we will start by examining the fundamental characteristics of a traditional forward-pumped EDFA, as illustrated in Fig. 2. This figure shows the variation within the EDFA of the pump power, the ASE power in both directions, and the internal gain coefficient of the forward-directed (small) signal. The gain accumulation within the EDFA can simply be calculated by integrating the internal gain coefficient along the fiber. The signal and pump wavelengths are $0.980 \mu \mathrm{m}$ and $1.534 \mu \mathrm{m}$, respectively, and the EDFA is Ge-doped. The fiber length is adjusted to maximum gain, that is, $51 \mathrm{~dB}$. A characteristic almost-symmetric shape of the small signal gain coefficient appears, showing a maximum near the middle of the amplifier. At the fiber ends, the internal gain coeffiient is depressed by the huge ASE power levels. Specifically, it is remarkable that the pump light leaving the fiber end is 


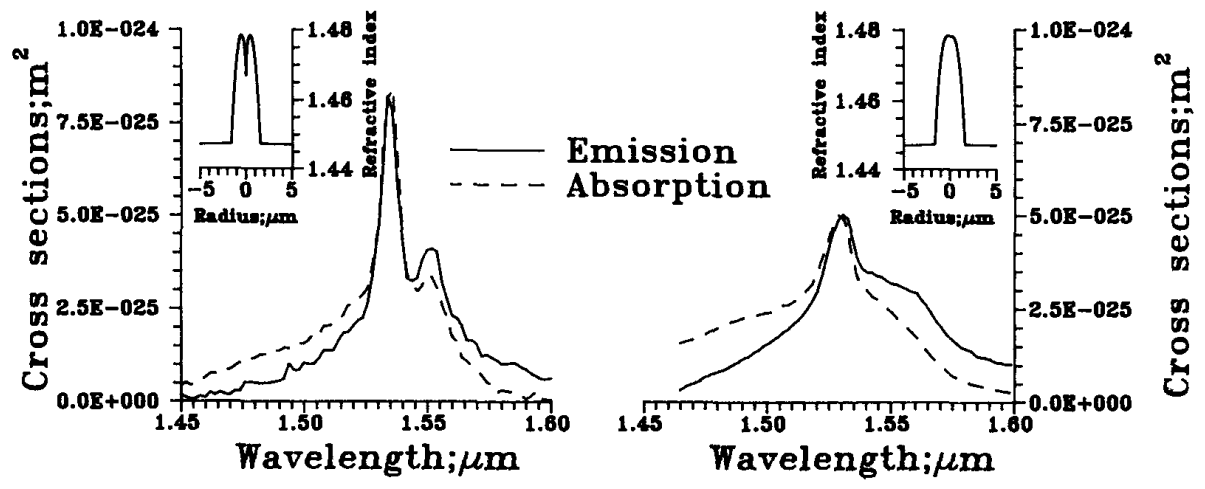

Fig. 1. Emission and absorption cross-section spectra and index profiles Left: Ge-doped EDFA. Right: Al-doped EDFA.
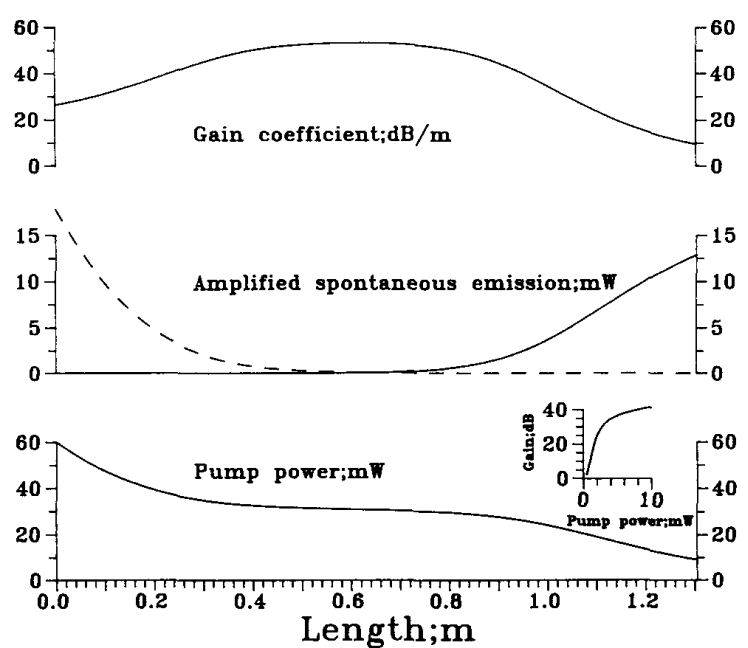

Fig. 2. Variation of gain coefficient, amplified spontaneous emission, and pump power inside a forward-pumped Ge-doped EDFA at optimum gain length. Solid curves: forward propagating. Dashed curves: backward propagating. Insert figure: Gain versus pump power. $\lambda_{p}=980 \mathrm{~nm}, \lambda_{s}=1534$ $\mathrm{nm}, P_{s}=-60 \mathrm{dBm}$.

far above pump threshold. As seen from the insert figure, the wasted pump power has a potential for driving an extra small signal amplifier with up to $40 \mathrm{~dB}$ gain. When we increase the length of the EDFA above its optimum gain length, the gain increase related to the extra length of fiber is weaker than the gain reduction in the input end of the fiber, caused by generation of a stronger backward-traveling ASE However, the latter can obviously be suppressed by insertion of an isolator within the EDFA, thereby making it possible to increase the gain above the optimum gain value.

Before we discuss the use of an isolator as an ASEattenuating component, it may be instructive to demonstrate that ASE reduction under certain circumstances occurs by itself when the signal wavelength is near the long wavelength emission peak at $1.55 \mu \mathrm{m}$. For this signal wavelength, and for a $0.98-\mu \mathrm{m}$ pump source, Fig. 3 shows that the bidirectional pumping scheme enhances the gain with $4 \mathrm{~dB}$ (Al-EDFA) and $10 \mathrm{~dB}$ (Ge-EDFA) compared to either the co- or the contradirectional pumping schemes. Similar calculations for signal wavelengths around the $1.53-\mu \mathrm{m}$ emission peak show, contrary to Fig. 3, that the gain is almost independent with respect to the degree of backward pumping. Still, the bidirectional pumping scheme is best, but the enhancement becomes less than $1 \mathrm{~dB}$. The huge gain enhancements of Fig. 3 can be understood from Fig. 4. It shows the internal variation of gain, ASE, and pump power for the Ge-EDFA at optimum gain length in a bidirectional pumped configuration using 0.98 $\mu \mathrm{m}$ sources and having signal wavelength at $1.55 \mu \mathrm{m}$. The length of the fiber is $8 \mathrm{~m}$, which is 6 times the length of the fiber in Fig. 2. It is seen that most pump power is consumed within the first $2 \mathrm{~m}$ from the ends, leaving $4 \mathrm{~m}$ in the middle where only ASE and signal are present. In this part of the EDFA the ASE acts as a pump source on the signal. This is possible only because the signal wavelength at $1.55 \mu \mathrm{m}$ is on the long wavelength side of the ASE peak at $1.53 \mu \mathrm{m}$. Thus the bidirectional pumping scheme may be viewed as three series-connected amplifiers, with the middle amplifier being pumped by forward and backward ASE generated in the surrounding amplifiers. However, only a very minor part of the 10-dB gain improvement is directly added by the extra gain from the middle amplifier which, as seen from Fig. 4, is very modest. The major part of the gain improvement is indirectly achieved through the strong 20-dB ASE attenuation in the middle part of the EDFA. This has the effect that fewer pump photons are spent to amplify the spontaneous emission, thereby increasing the pump efficiency.

Even though ASE attenuation is strongly present in this situation, it is still advantageous to implement an isolator. The reason is that the ASE attenuation in Fig. 4 occurs in the middle of the fiber, while the optimum placement is found to be to be close to the signal input end of the EDFA. The internal variation of gain, ASE, and pump power for the situation with an optimally placed isolator within the Ge-EDFA at optimum gain length is shown in Fig. 5. The isolator has a loss of $2.3 \mathrm{~dB}$ in codirection and $41.6 \mathrm{~dB}$ in counterdirection. Compared with Fig. 4, the total length has increased $40 \%$, and a pronounced asymmetric variation appears. The gain coefficient has increased $30 \%$ in the input end of the EDFA because the backward-traveling ASE is attenuated to $15 \%$ of 


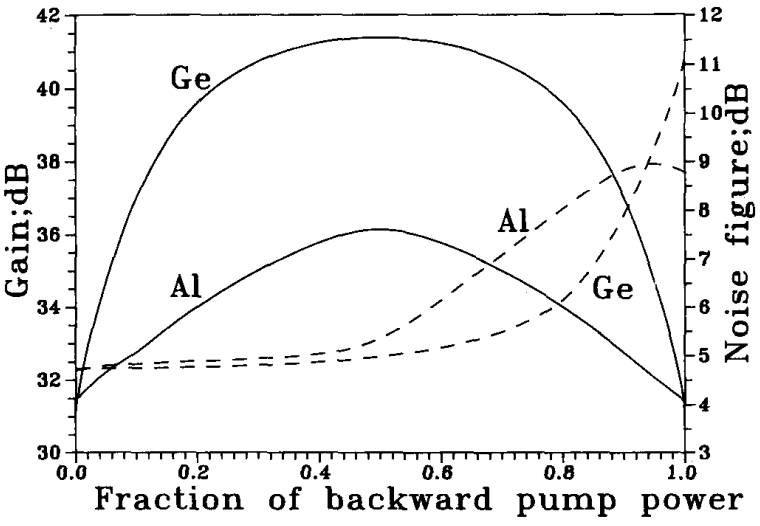

Fig. 3. Gain and noise figures for Ge-doped EDFA and Al-doped EDFA against fraction between backward pump power and total pump power. Right axis curves: dashed. Left axis curves: solid. $\lambda_{p}=980 \mathrm{~nm}, \lambda_{s}=1552 \mathrm{~nm}$, $P_{p}=60 \mathrm{~mW}, P_{s}=-60 \mathrm{dBm}$.
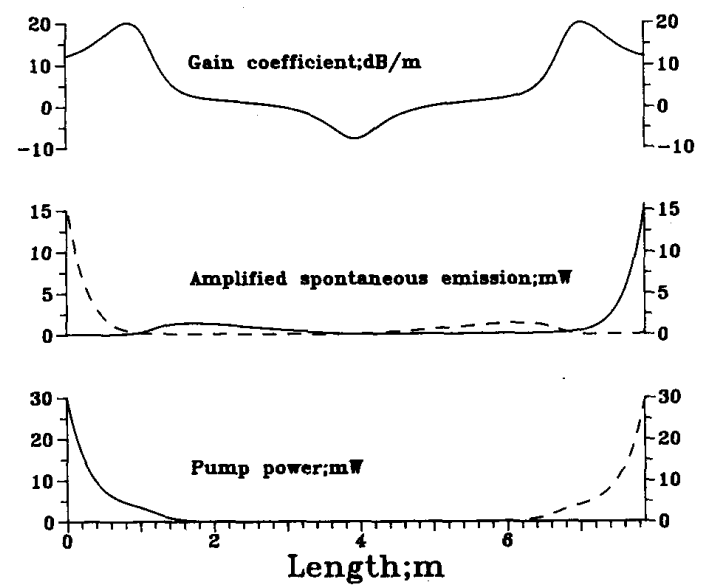

Fig. 4. Variation of gain coefficient, amplified spontaneous emission, and pump power inside a bidirectional pump Ge-doped EDFA at optimum gain length. Solid curves: forward-propagating pump fraction. Dashed curves: backward-propagating pump fraction. $\lambda_{p}=980 \mathrm{~nm}, \lambda_{s}=1552 \mathrm{~nm}$, $P_{p}=60 \mathrm{~mW}, P_{s}=-60 \mathrm{dBm}$.

the situation in Fig. 4. This gain increase is also advantageous because the noise accumulates more slowly when the inversion becomes more complete. In the actual situation the noise figure is very close to the $3-\mathrm{dB}$ limit and $1.5 \mathrm{~dB}$ better than in the situation without an isolator. It should also be noticed from Fig. 5 that the isolator divides the EDFA into two EDFA pieces, each having significant gain. Therefore, another advantage is that the EDFA with an implemented isolator becomes resistant against lasing.

After this survey of the main differences between the ASE buildup for different source wavelengths and co-doping materials, we now focus mostly on the $1.47-\mu \mathrm{m}$ pumping of the $1.55-\mu \mathrm{m}$ signal in an Al-doped EDFA. These parameters are chosen in agreement with the measurements presented in the last part of the paper. Unless otherwise stated, the amount of pump power is $60 \mathrm{~mW}$ and the signal input power is $-60 \mathrm{dBm}$. However, the other relevant pump band
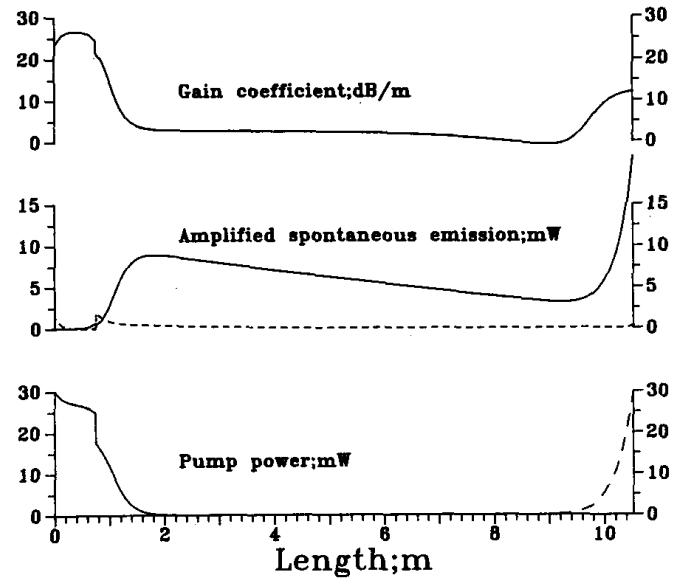

Fig. 5. Variation of gain coefficient, amplified spontaneous emission, and pump power inside a bidirectional pumped Ge-doped EDFA at optimum gain length and with a WDM-isolator (see Section III) implemented at optimum placement. Solid curves: forward propagation. Dashed curves: backward propagation. $\lambda_{p}=980 \mathrm{~nm}, \lambda_{s}=1552 \mathrm{~nm}, P_{p}=60 \mathrm{~mW}, P_{s}=-60 \mathrm{dBm}$.

at $0.980 \mu \mathrm{m}$, signals at the fluorescence peak at $1.530 \mu \mathrm{m}$, and Ge-doped EDFA's are also included in some simulations for exemplifying major differences between the choice of wavelengths or EDFA type.

\section{ISOLATOR LOCATION FOR DifFERENT PUMP CONFIGURATIONS}

A main difference exists in the improvement of the performance that can be obtained by using the isolator within the fiber when pumping at the two most relevant pump wavelength bands for EDFA operation, namely the $0.98-\mu \mathrm{m}$ and the 1.47 $\mu \mathrm{m}$ band. As measurements of the isolator insertion loss for the forward propagating light show variations of several tens of decibels within the considered spectral range from $0.98 \mu \mathrm{m}$ to $1.55 \mu \mathrm{m}$, the use of WDM couplers will be suggested in connection with a $0.98-\mu \mathrm{m}$ pump source.

Let us first consider the $1.47-\mu \mathrm{m}$ pump source. The pump wavelength is that close to the signal that the isolator will decrease the co- and counterpropagating pump light in a way similar to the decrease for the co- and counterpropagating signal. Improvements can therefore hardly be obtained for counterpumped EDFA's. In the case of the $0.98-\mu \mathrm{m}$ pump source, high loss values are measured for both directions. These are $37.8 \mathrm{~dB}$ for the copropagating and $27.1 \mathrm{~dB}$ for the counterpropagating pump, leaving only the bidirectional pumping scheme as a potential configuration for isolator optimization. However, by using two WDM couplers (Fig. 6 ), it is possible to guide the pump past the isolator with two times the insertion loss of the WDM coupler, which has been measured to $1.5 \mathrm{~dB}$ in each direction. We will denote this configuration as a WDM isolator, and the former as a pure isolator. The signal and the ASE will still be guided through the isolator with a loss of $2.3 \mathrm{~dB}$ in the codirection and $41.6 \mathrm{~dB}$ in the counterdirection, which includes the extra loss from the WDM couplers. All considered losses include splice losses. 


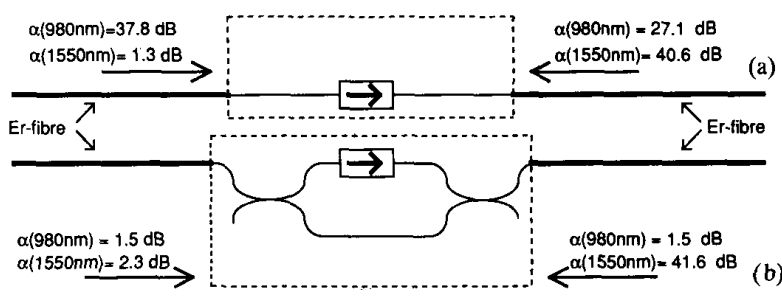

Fig. 6. Isolator configurations. (a) An isolator directly inserted in the erbium-doped fiber will be denoted as the "pure isolator scheme." (b) The pump by-pass coupling around the isolator will be denoted as the "WDM isolator."

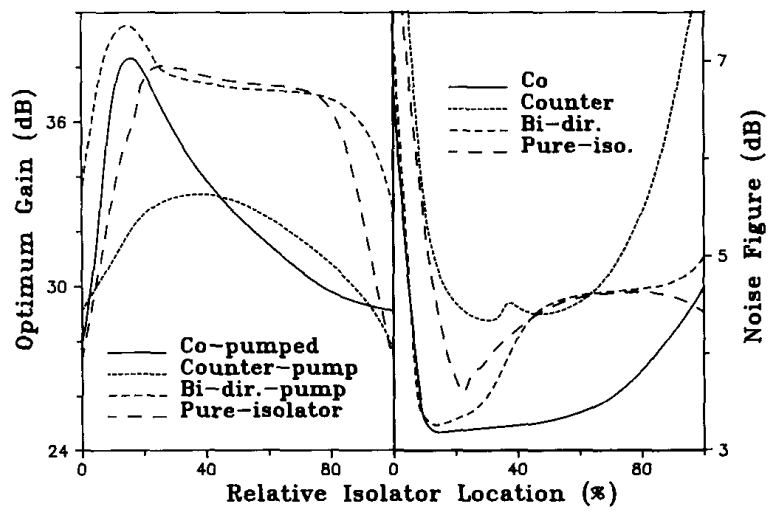

Fig. 7. Optimum gain and the corresponding noise figure versus the relative isolator location in percent. Curves are shown for co-, counter-, and bidirectional pumped amplifiers with a WDM isolator inserted. An additional curve shows the results for the latter scheme when using a pure isolator. $\lambda_{p}=0.98 \mu \mathrm{m}, \lambda_{s}=1.55 \mu \mathrm{m}, P_{s}=-60 \mathrm{dBm}$, and $P_{p}=60 \mathrm{~mW}$.

As shown in Fig. 3, the fraction between the backward- and forward-travelling pumps plays a significant role in the performance of the EDFA. This is also the case when an isolator is implemented (Fig. 7). Here the performances of copumped, counterpumped, and bidirectional-pumped configurations are shown for various placements of a pure isolator or a WDM isolator (for $\lambda_{p}=0.98 \mu \mathrm{m}$ ). The signal wavelength is $1.55 \mu \mathrm{m}$. The optimum isolator placement is seen to be between $15 \%$ and $37 \%$ of the total EDFA length.

Comparing the reference gain for similar EDFA's in Fig. 3 , it is seen that the copropagating pumping scheme benefits the largest improvements, while the global best performance is achieved with the bidirectional pump configuration including a WDM isolator. Looking at the $0.98-\mu \mathrm{m}$ bidirectional-pumped pure isolator scheme, it is seen that isolator placement more than $25 \%$ from the middle results in poor noise and gain performance. Further, the optimum placement in the middle yields only $1.5-\mathrm{dB}$ lower gain than the more complicated WDM-isolator scheme.

Comparing the isolator locations optimized with respect to noise with those optimized with respect to gain, it is seen that the former is a few percent closer to the front end of the EDFA. However, the difference is not significant; therefore, optimizing the bidirectional pumping scheme with respect to gain only results in $0.3-\mathrm{dB}$ noise figure deterioration. The best performances are obtained by co- and bidirectional pumping and the global lowest noise figure of $3.2 \mathrm{~dB}$ is very close to the theoretical lower limit when pumped at $0.98 \mu \mathrm{m}$. Omitting the pump bypass coupling of the bidirectional scheme, it is seen that a deterioration of $0.6 \mathrm{~dB}$ in the noise figure adds to the $1.5 \mathrm{~dB}$ gain reduction, for the pure isolator

\section{Performance DePendence UPON THE WAVELENGTH CHOICE}

The quantities of corresponding gain and noise figure improvements also much depend on the choice of pump and signal wavelength due to their different influence upon ASE generation. Therefore, the influence of the isolator insertion is investigated for different signal wavelengths when pumping at $0.980 \mu \mathrm{m}$ or $1.470 \mu \mathrm{m}$. Calculations are made for both an $\mathrm{Al}-$ and a Ge-co-doped erbium fiber. For the latter, signal wavelengths corresponding to the two local fluorescence maxima at $1.534 \mu \mathrm{m}$ and $1.552 \mu \mathrm{m}$ are assumed, while for the former a signal at the fluorescence maximum at $1.530 \mu \mathrm{m}$ and a signal in the low loss window at $1.550 \mu \mathrm{m}$ are considered.

Concerning the noise figure, it is seen from Fig. 8 that a combination of $1.47-\mu \mathrm{m}$ pump and $1.55-\mu \mathrm{m}$ signal will benefit most from the insertion of an isolator in an aluminum co-doped EDFA. A decrease of $2.8 \mathrm{~dB}$ is achieved. However, the favorite configuration with respect to the lowest attainable noise figure for a high-gain amplifier is the $0.98-\mu \mathrm{m}$ pumping of the 1.53 $\mu \mathrm{m}$ signal yielding a noise figure of less than $3.2 \mathrm{~dB}$, which is very close the theoretical $3.0-\mathrm{dB}$ lower limit. The largest gain improvement, $4.9 \mathrm{~dB}$, is obtained for $0.98-\mu \mathrm{m}$ and $1.47-\mu \mathrm{m}$ pumping of the $1.53-\mu \mathrm{m}$ signal, and in addition the overall highest gain of $50.5 \mathrm{~dB}$ is calculated for the former.

For the germanium fiber the isolator increases the gain by $10 \mathrm{~dB}$ and improves the noise figure by $6 \mathrm{~dB}$ for the case with the $1.47-\mu \mathrm{m}$ pump and $1.53-\mu \mathrm{m}$ signal. The best noise and gain performances are obtained with $0.98-\mu \mathrm{m}$ pump, where a signal wavelength at $1.53 \mu \mathrm{m}$ gives $58-\mathrm{dB}$ gain and $3.1-\mathrm{dB}$ noise figure. For the $1.55-\mu \mathrm{m}$ signal, the absolute values are $48-\mathrm{dB}$ gain and $3.3 \mathrm{~dB}$ for the noise figure.

Recently it has been reported [10] that pumping at the absorption peak does not give the highest gain in the $0.98-\mu \mathrm{m}$ band when signal wavelengths around $1.55 \mu \mathrm{m}$ are considered. This gain hole spectrum is illustrated as a dashed line in Fig. 9, which shows simultaneous values of gain and noise figure for gain-optimized fiber lengths versus the pump wavelength. A copropagating pump configuration is assumed with all data similar to the above-mentioned, except for the pump wavelength. A 1.1-dB gain hole is observed when pumping at $0.980 \mu \mathrm{m}$ instead of detuning to the gain shoulders around $0.960 \mu \mathrm{m}$ or $0.996 \mu \mathrm{m}$, but still the lowest noise figure is achieved at $0.980 \mu \mathrm{m}$.

As the gain hole phenomenon is caused by ASE saturation of the amplifier when the emission cross section exceeds a certain level, the hole can be eliminated by insertion of the ASE attenuating isolator in the fiber. Calculations with the isolator at the optimum location give the solid lines showing that both maximum gain and the minimum noise figure in 


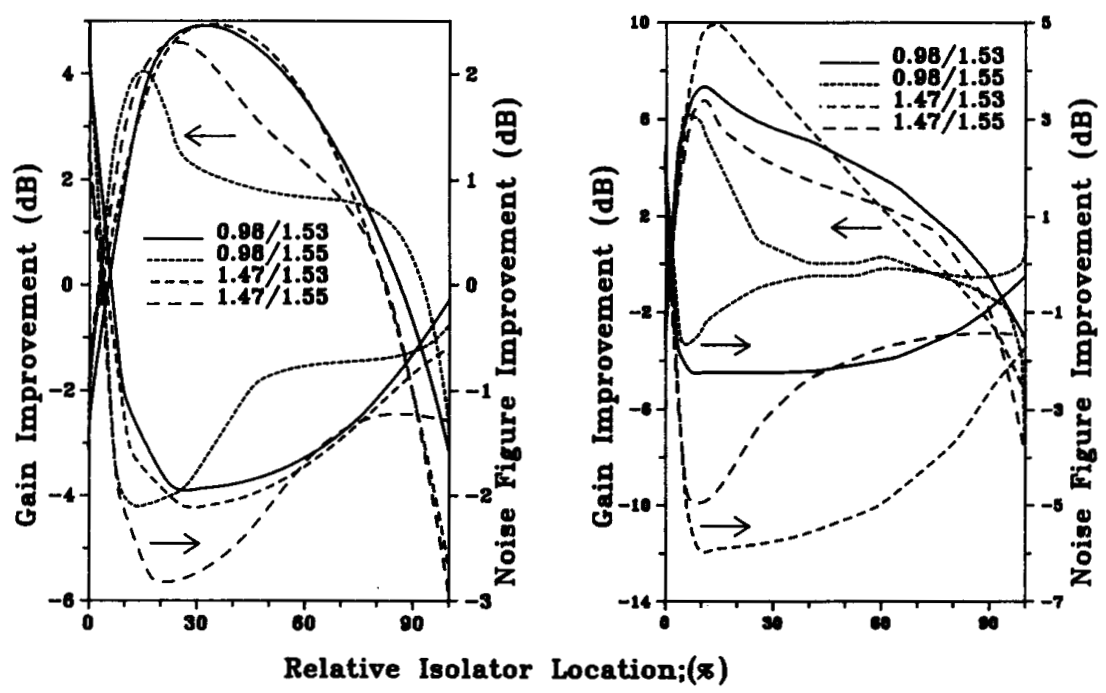

Fig. 8. Gain and noise figure improvements versus the relative isolator location in percent for four different combinations of pump and signal wavelengths. Left: Results for an Al-doped EDFA. Right: Results for a Ge-doped EDFA. A bi-directional pumping scheme is considered.

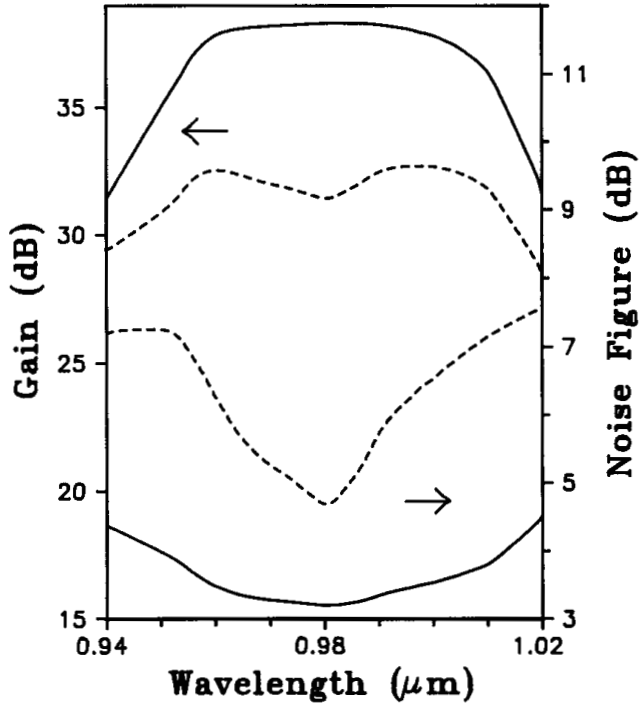

Fig. 9. Optimum gain and corresponding noise figure for different pump wavelengths in the $0.98-\mu \mathrm{m}$ band. Dotted lines: Results on the reference fiber. Solid lines: Results with an inserted WDM isolator. $\lambda_{s}=1.55 \mu \mathrm{m}$, $P_{s}=-60 \mathrm{dBm}$, and $P_{p}=60 \mathrm{~mW}$.

this case are obtained for pumping at the absorption peak at $0.98 \mu \mathrm{m}$.

\section{VARIATION OF THE POWER LEVELS}

For the above-mentioned pump configurations, the optimum isolator location within the erbium fiber was analyzed for EDFA's operated in the small signal regime and using a fixed pump power of $60 \mathrm{~mW}$. However, when changing either the signal or the pump power, the ASE buildup and thereby also the optimum isolator location will change. Calculations of the gain and noise figure for increasing input signal power in the erbium fiber are shown in Fig. 10. Signal wavelengths at

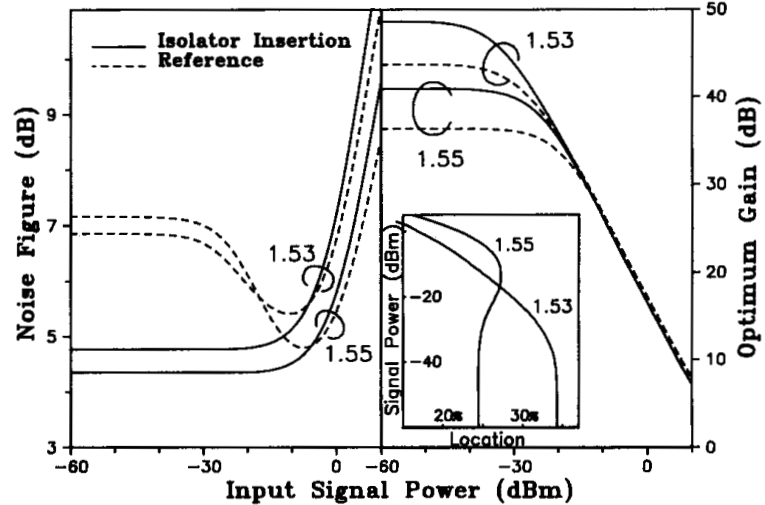

Fig. 10. Optimum gain and corresponding noise figure versus the input signal power to the erbium-doped fiber. Both signals at $1.53 \mu \mathrm{m}$ and at $1.55 \mu \mathrm{m}$ are considered. Dotted lines: results of the reference fiber. Solid lines: results when a pure isolator is considered. Insert: Optimum isolator location versus input signal power. $\lambda_{p}=1.47 \mu \mathrm{m}$ and $P_{p}=60 \mathrm{~mW}$.

$1.53 \mu \mathrm{m}$ and $1.55 \mu \mathrm{m}$ are considered together with $60 \mathrm{~mW}$ of launched pump power at $1.47 \mu \mathrm{m}$. The dashed reference lines illustrate the performance of a gain-optimized fiber without an isolator, while the solid lines show the results for a gain-optimized fiber with an isolator inserted at its optimum location. The latter is denoted as the test fiber.

For both signal wavelengths an input signal power below $-5 \mathrm{dBm}$ allows for a net improvement in the noise figure by insertion of the isolator, while a minor noise figure increase relative to the reference arises for higher power levels. Improvements of $2.1 \mathrm{~dB}$ and $2.8 \mathrm{~dB}$ are achieved at $1.53 \mu \mathrm{m}$ and $1.55 \mu \mathrm{m}$, respectively, in the small signal regime where the large amount of ASE deteriorates the population inversion of the reference fiber. For increasing power levels the reference noise figure will improve, as the signal saturation rather than the ASE will determine the population inversion. However, 


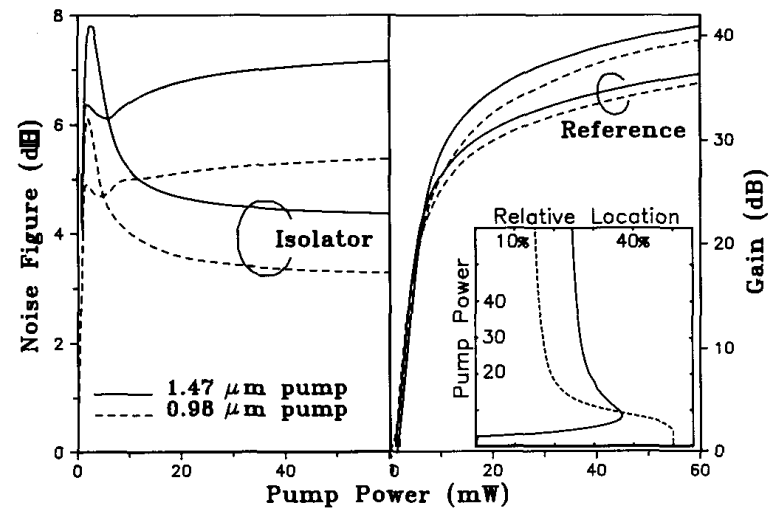

Fig. 11. Optimum gain and corresponding noise figure versus the pump power into the erbium-doped fiber. Calculations are made both with and without an inserted isolator. Dotted lines: results for $0.98-\mu \mathrm{m}$ pumping. Solid lines: results for $1.48-\mu \mathrm{m}$ pumping. Insert: Optimum isolator location versus pump power. $\lambda_{s}=1.55 \mu \mathrm{m}$ and $P_{s}=-60 \mathrm{dBm}$.

in the large signal regime the signal saturation alone will be determinative; hence, there is no improvement by using the isolator and both the reference as well as the test fiber noise figures increase rapidly.

Gain improvements up to $4.9 \mathrm{~dB}$ for $1.53-\mu \mathrm{m}$ and $4.6 \mathrm{~dB}$ for $1.55-\mu \mathrm{m}$ signals are achieved for signal powers below the $3-\mathrm{dB}$ saturation point, which are $-28 \mathrm{dBm}$ and $-18 \mathrm{dBm}$, respectively. However, it is seen that the input signal has to be approximately $10 \mathrm{~dB}$ lower than the 3 - $\mathrm{dB}$ saturation point to allow for gains within a few tenths of a decibel from the value in the extreme small signal region. In the large signal regime the isolator insertion loss has a more weighty influence upon the amplifier performance than the moderate profit of ASE reduction, resulting in a minor gain decrease.

The optimum isolator location within the fiber is illustrated versus the input signal power in the lower right part of Fig. 10, showing a large difference between the two considered signal wavelengths. In the large signal regime a signal wavelength at $1.53 \mu \mathrm{m}$ gives a more noisy amplifier than the one at $1.55 \mu \mathrm{m}$. Therefore, the $1.53-\mu \mathrm{m}$ signal leads to a shorter optimum location than the one at $1.55 \mu \mathrm{m}$. However, for decreasing signal power the ASE builds up much stronger for the 1.55$\mu \mathrm{m}$ signal, resulting in shorter isolator locations when using this wavelength. In the range where the signal saturation is determinative of the population inversion, the relative isolator location is seen to increase for decreasing signal powers. However, for signals below the 3-dB saturation point, the location remains unchanged at $34 \%$ and $24 \%$ for $1.53-\mu \mathrm{m}$ and $1.55-\mu \mathrm{m}$ signals, respectively.

The influence of pump power variations upon the amplifier performance of the $1.55-\mu \mathrm{m}$ signal is investigated for pump wavelengths at both $0.98 \mu \mathrm{m}$ and $1.47 \mu \mathrm{m}$ when using the pump bypass coupling for the former. In Fig. 11 calculations are represented for a signal input power level at $-60 \mathrm{dBm}$.

Pump power levels sufficient to achieve a $20-\mathrm{dB}$ gain by $0.98 \mu \mathrm{m}$ pumping and $24 \mathrm{~dB}$ by $1.47-\mu \mathrm{m}$ pumping are required before the use of the considered isolator, with realistic insertion losses, gives improvements in gain and noise figure

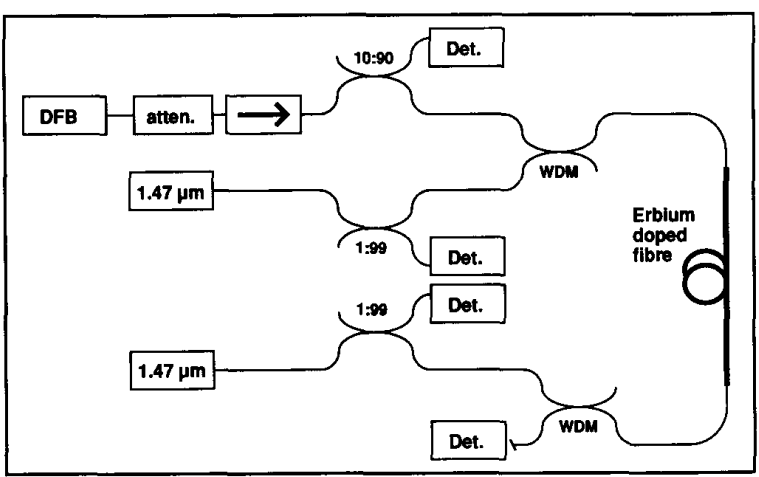

Fig. 12. Experimental setup.

performance. However, above this limit both the gain and noise figure improvements increase with increasing pump power. For the latter this clearly appears from the curves on the left part of the figure as the reference increases for increasing pump due to a faster increase in ASE than in the gain. By insertion of the isolator, the course is opposite, as the gain will increase more rapidly than the ASE. Use of an ideal (lossless) isolator would be beneficial at any gain level.

The optimum isolator location as represented in the lower right corner of Fig. 11 shows large changes with pump power. In the uninteresting range for small pump powers, where the reference yields a better performance than use of the isolator, the optimum location goes toward $50 \%$ for $0.98-\mu \mathrm{m}$ pumping and toward $0 \%$ for $1.47-\mu \mathrm{m}$ pumping. The former is due to equal attenuation of both forward and backward pump in the bypass coupling. It makes this position optimum as least pump is attenuated by the isolator when placed in the middle of the fiber. At 1.47 the attenuation of the backward pump is 39.3 $\mathrm{dB}$ larger than for forward, making a position in the beginning of the fiber optimum. However, for power levels well above the previously mentioned application limits, it is seen that the optimum position remains unchanged at $15 \%$ when pumping at $0.98 \mu \mathrm{m}$ and $24 \%$ at $1.47 \mu \mathrm{m}$.

\section{EXPERIMENTAL SetuP}

The experimental setup for evaluating the gain characteristics is shown in Fig. 12. A bidirectional pumping configuration is assumed, using two InGaAsP/InP lasers, each emitting at $1.47 \mu \mathrm{m}$ and capable of delivering up to a total of $70 \mathrm{~mW}$ of launched pump power into the erbium-doped fiber. In each of the pump branches, the laser is followed by a $1: 99$ coupler used for measure of the pump power reference. The output arm of this coupler is spliced to the WDM coupler located at each end of the EDFA. All free fiber ends in these as well as in the other couplers in the setup are cleaved in angles of $10^{\circ}$ to prevent unwanted reflections.

The modulated signal light at $1.55 \mu \mathrm{m}$ originates from a temperature-tuned DFB laser and the signal power is adjusted by an attenuator to a level of $-40 \mathrm{~dB}$ at the input end of the EDFA. An isolator is inserted to prevent backward-travelling ASE from reaching the signal laser. To allow for a reference measurement of the signal, a $10: 90$ coupler is inserted in the 


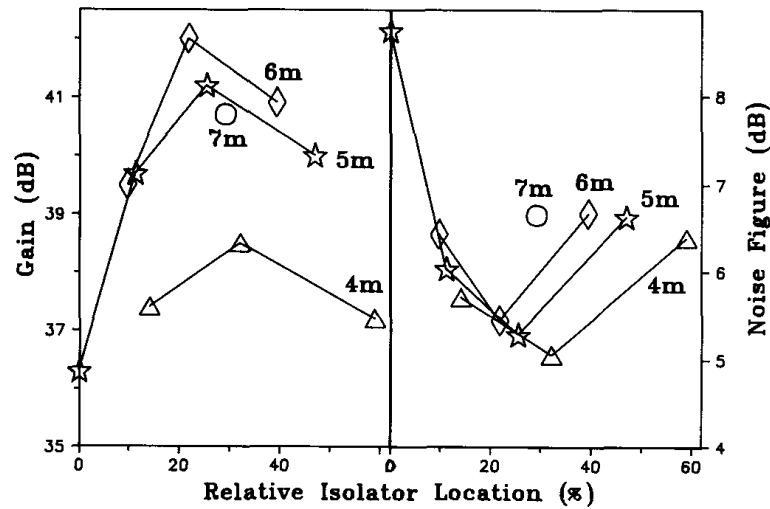

Fig. 13. Measured gain and the corresponding noise figure versus the relative isolator location. Measurements are shown at four different fiber lengths: $4 \mathrm{~m}$, $5 \mathrm{~m}, 6 \mathrm{~m}, 7 \mathrm{~m} . \lambda_{p}=1.47 \mu \mathrm{m}, \lambda_{s}=1.55 \mu \mathrm{m}, P_{s}=-40 \mathrm{dBm}$, and $P_{p}=60$ $\mathrm{mW}$.

signal way before the WDM coupler at the input end of the amplifier. On the output end of the EDFA the signal is guided through the WDM coupler before it is recorded by use of a lock-in amplifier with an integrating sphere detector.

The setup for evaluating the noise figure is somewhat similar, as it is determined from corresponding measurements of gain $G$ and ASE power $P_{\mathrm{ASE}}$, in a bandwidth $\Delta v$ around the signal frequency $v$ by the equation [8]:

$$
\mathrm{NF}=\frac{1}{G} \cdot\left(\frac{P_{\mathrm{ASE}}}{\Delta v \cdot h \cdot v}+1\right)
$$

where $h$ is Planck's constant. The shape of the ASE spectrum is recorded in $0.1-\mathrm{nm}$ steps on a double monochromator, and by integrating the area under the curve in respective a bandwidth $\Delta v$ around the signal, and in the whole spectral wavelength range, a relative measure of the ASE within the bandwidth is obtained. $P_{\mathrm{ASE}}$ is determined by scaling this fraction with the total amount of ASE power, measured by a wavelength-calibrated power meter with an integrating sphere detector. The double monochromator is used as it allows for a higher dynamic range, which is important for measuring the ASE level close to the signal wavelengths.

\section{MEASUREMENTS}

For a launched pump power level at $60 \mathrm{~mW}$ from the above mentioned bidirectional pumped scheme and with an input signal power at $-40 \mathrm{dBm}$, the length of the erbiumdoped fiber is gain-optimized, yielding a maximum gain of $37.9 \mathrm{~dB}$ and a corresponding noise figure of $7.4 \mathrm{~dB}$. These numerical quantities measured on the approximately 5-m-long reference fiber are denoted below as the reference gain and the reference noise figure, respectively. Further, the erbium-doped fiber with an isolator inserted within its length is referred to as the test fiber. Now, by insertion of the isolator at a relative location between $0 \%$ and $60 \%$ of the total fiber length, which is varied between 0.8 and 1.4 times the reference fiber length, the measured gains and the corresponding noise figures are represented in Fig. 13.

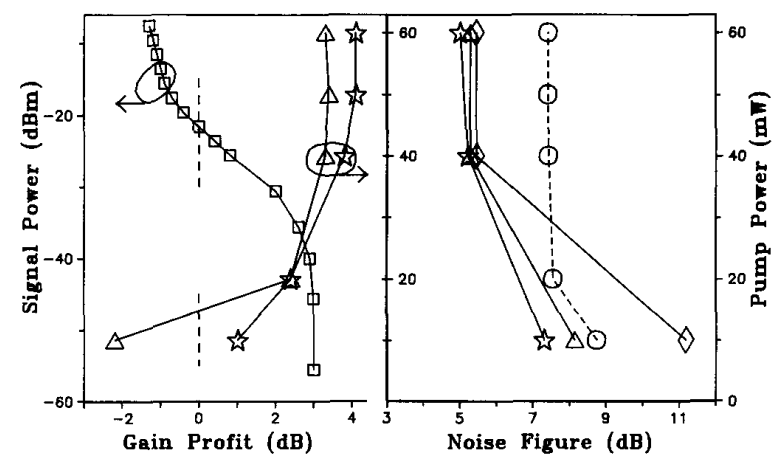

Fig. 14. Left $\square$ : Measured gain profit relative to the reference fiber for signal power variations into a 7 -m-long fiber. $\triangle$ : Profits for pump power variations are shown for an isolator inserted in the reference fiber. The stars shows the best results, selected among 11 different fiber lengths. Right $\mathrm{o}$ : Noise figure versus pump power measured on the reference fiber, $\diamond$ : fiber length that is gain optimum for a $60-\mathrm{mW}$ pump. Stars and triangles are explained above.

The predicted optimum isolator location within the first third of the fiber is obviously verified by the measurements. An overall highest gain of $42.0 \mathrm{~dB}$ is achieved for an isolator placed at $22 \%$ of a fiber that is approximately $20 \%$ longer than the reference. The overall lowest noise figure at $5.0 \mathrm{~dB}$ is, as expected, measured at the shortest fiber length. However, the noise figure corresponding to the gain optimized isolator configuration is only four tenths of a decibel higher. The measurements clearly show how important a correct position of the isolator is. Consider a fiber length similar to the reference, a gain reduction of $4.9 \mathrm{~dB}$ and a corresponding noise figure deterioration of $3.5 \mathrm{~dB}$ are caused by moving the isolator from its optimum location to the signal input end.

For given pump and input signal powers, the gain profit denotes that part of the gain measured on the test fiber, which exceeds the gain of the reference fiber measured for corresponding signal and pump powers. The influence of variation in the input signal power upon the gain profit is illustrated in the left part of Fig. 14. As the test fiber is slightly above its optimum length in the small signal regime and the relative isolator location is some percentage larger than its optimum placement, only $3-\mathrm{dB}$ gain profit is obtained for $P s=-40 \mathrm{dBm}$.

The interesting region in which a positive gain profit is obtained is observed to be limited by a signal power of -22 $\mathrm{dBm}$. However, the optimum length of the test fiber $L_{\text {test,opt }}$ decreases more rapidly than the optimum length of a fiber without an isolator, $L_{\mathrm{opt}}$, for increasing signal power. As both test and reference fiber length are unchanged in the considered variation process, a length-dependent decrease in the gain profit will appear for increasing $P s$. This influence is estimated to extend the positive gain profit range with some few decibels.

The influences of pump power changes are also seen in Fig. 14, for power variations between 10 and $60 \mathrm{~mW}$. The triangles correspond to measurements with an isolator inserted in the reference fiber, while the stars show the optimum measurements selected among 11 different fiber lengths and isolator locations. It is seen from the left part of the figure that 


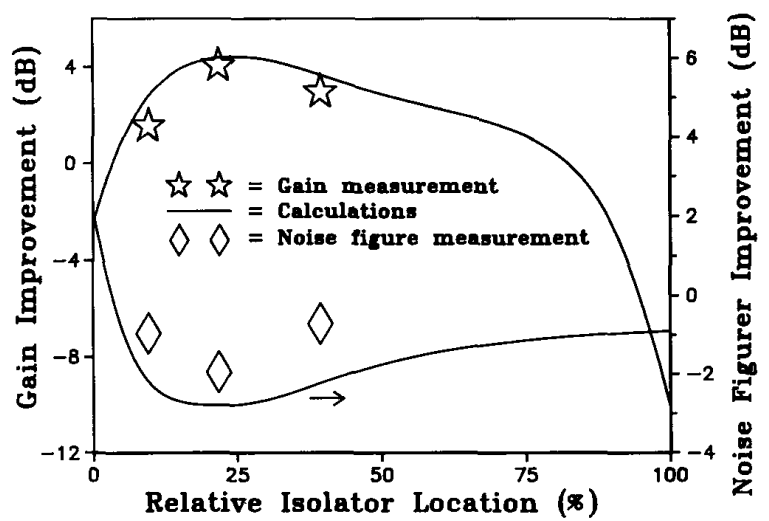

Fig. 15. Measured gain improvements relative to the reference fiber shown as stars and the corresponding noise figure illustrated as diamonds, versus the relative isolator location. Corresponding calculations are represented as solid lines. $\lambda_{p}=1.47 \mu \mathrm{m}, \lambda_{s}=1.55 \mu \mathrm{m}, P_{3}=-40 \mathrm{dBm}$, and $P_{p}=60 \mathrm{~mW}$.

more than two decibels of gain profit are obtained for pump powers above $20 \mathrm{~mW}$ simply by insertion of an isolator in the gain-optimized reference fiber. By an optimization of the fiber length of the new configurations, a gain profit between 3.8 and $4.1 \mathrm{~dB}$ is obtained for pump power levels above $40 \mathrm{~mW}$.

The correlation between the noise figure and the launched pump power is illustrated on the right part of Fig. 14. The circles connected by a dashed line represent measurements on the reference fiber. An almost constant noise figure of $7.4 \mathrm{~dB}$ is obtained for $P p$ above $20 \mathrm{~mW}$. An improvement for all pump power levels is observed when the isolator is inserted in the reference fiber (solid line with triangles), even though the corresponding gain for $10 \mathrm{~mW}$ power is more than $2 \mathrm{~dB}$ lower than the reference. This is due to the even larger decrease of the spontaneous emission. By increasing the length of the test fiber to the length that is gain-optimum for $60-\mathrm{mW}$ pump, measurements shown as diamonds are obtained. The same low noise figure is seen for high pump, but the length-dependent gain profit decrease for small pump powers will increase the noise figure.

Finally, the gain and noise figure improvements of the 6-mlong test fiber relative to the reference fiber are shown in Fig. 15. The measured data are represented in comparison with calculations of the performance improvements for different placements of the isolator in a fiber having a fixed length. Corresponding to the measurements, this fixed length is chosen as the optimum length when the isolator is inserted at its optimum location. Numerous measured data for the considered fiber, similar to those in the previous sections, are used as input parameters to the model. The absorption and emission cross sections for an ordinary aluminum co-doped fiber are considered as they show good agreement with the test fiber [11], and the measured index profile is found to approach an $\alpha=3$ profile.

The magnitude as well as the curve progress for the calculated gain improvements versus the isolator location show very high agreement with the measurements in the range that has been experimentally investigated. The largest difference is approximately $1 \mathrm{~dB}$ and in both cases the optimum relative isolator location is determined to be near $20 \%$ of the total fiber length. Less than 1.5-dB difference is observed between the calculated and measured noise figure improvements. The optimum isolator position with respect to the noise figure is both experimentally as well as theoretically determined to be near $20 \%$ of the total fiber length.

\section{CONCLUSION}

The insertion of an isolator within the length of an erbiumdoped fiber has been shown to enable near-quantum-limited operation of very-high-gain amplifiers. When pumping at $1.47 \mu \mathrm{m}$ the isolator can be directly installed in the fiber, as only a minor insertion loss is present at this wavelength. For $0.98-\mu \mathrm{m}$ pumping a large insertion loss will deteriorate the pump dramatically, but a bypass coupling of the pump has been shown to solve this problem efficiently.

As in the case without an ASE attenuation component, the best pump configuration with respect to high gain and low noise is the bidirectional one. Experiments have yielded improvements of up to $4.1 \mathrm{~dB}$ and $2.0 \mathrm{~dB}$ in the gain and noise figures, respectively, by using the isolator in such a scheme. Still, even larger improvements are predicted for the copropagating configuration. Depending on the chosen pumping scheme the optimum isolator location varies between $15 \%$ and $37 \%$ of the total fiber length when pumping the $1.55-\mu \mathrm{m}$ signal at $0.98 \mu \mathrm{m}$.

The combination of a $0.98-\mu \mathrm{m}$ pump and a $1.53-\mu \mathrm{m}$ signal wavelength is the best choice with respect to noise figure. For the gain-optimized fiber a noise figure only $0.2 \mathrm{~dB}$ above the $3.0-\mathrm{dB}$ quantum limit is calculated. Still, it is the 1.47 $\mu \mathrm{m}$ pumped $1.55-\mu \mathrm{m}$ signal that benefits most of the isolator insertion. Combination of the considered pump and signal wavelength yields optimum isolator locations between $15 \%$ and $36 \%$ of the fiber length.

The amplifier improvements by using the isolator exist in the whole input signal power range where ASE is determinative of the population inversion. Gain improvements can be obtained for input signal powers below the $3-\mathrm{dB}$ saturation point and noise figure improvements for signal powers below $-5 \mathrm{dBm}$. The optimum isolator location increases for decreasing signal power and becomes unchanged in the small signal regime where the signal at $1.53 \mu \mathrm{m}$ gives ten percentage points larger isolator locations than signals at $1.55 \mu \mathrm{m}$. Similarly, the 0.98 $\mu \mathrm{m}$ pump yields optimum locations some ten percentage points shorter than $1.47-\mu \mathrm{m}$ pumps.

\section{ACKNOWLEDGMENT}

The authors thank LYCOM A/S for the supply of the Erdoped fiber and Dr. E. Nicolaisen for fruitful discussions.

\section{REFERENCES}

[1] M. Shimizu, M. Yamada, M. Horiguchi, T. Takeshita, and M. Okayasu, "Erbium-doped fibre amplifiers with an extremely high gain coefficient of $11.0 \mathrm{~dB} / \mathrm{mW}$," Electron. Lett., vol. 26, no. 20, pp. 1641-1643, 1990.

[2] S. L. Hansen, K. Dybdal, and C. C. Larsen, "Upper gain limit in Erdoped fiber amplifiers due to internal Rayleigh backscattering," Proc. Conf. Optical Fiber Commun. '92, 1992, p. 68.

[3] R. Olshansky, "Noise figure for erbium-doped optical fibre amplifiers," Electron. Lett., vol. 24, no. 22, pp. 1363-1365, 1988. 
[4] R. I. Laming and D. N. Payne, "Noise characteristics of erbium-doped fiber amplifier pumped at $980 \mathrm{~nm}$," Photon. Technol. Lett., vol. 2, no. 6, pp. 418-421, 1990.

[5] J. H. Povlsen, A. Bjarklev, O. Lumholt, H. Vendeltorp-Pommer, and K. Rottwitt, "Optimizing gain and noise performance of EDFA's with insertion of a filter or an isolator," Proc. SPIE OE/FIBERS '91, 1991.

[6] Y. Aoki, T. Saito, K. Fukagai, Y. Sunohara, S. Ishikawa, and S. Fujita, "Low noise and high saturation output power erbium-doped fiber amplifiers pumped with $0.98 \mu \mathrm{m}$ and $1.48 \mu \mathrm{m}$ LDs for longdistance optical communication," Proc. 17th ECOC '91, Paris, 1991, pp. $585-588$.

[7] C. R. Giles and D. J. Di Giovanni, "Dynamic gain equalization in two-stage fiber amplifiers," Photon. Technol. Lett., vol. 2, no. 12, pp. $866-868,1990$

[8] B. Pedersen, A. Bjarklev, J. H. Povlsen, K. Dybdal, and C. C. Larsen, "The design of erbium-doped fiber amplifiers," J. Lightwave Technol., vol. 9, no. 9, pp. 1105-1112, 1991.

[9] B. Pedersen, A. Bjarklev, O. Lumholt, and J. H. Povlsen, "Detailed design analysis of erbium doped fiber amplifiers," Photon. Technol. Lett., vol. 3, no. 6, pp. 548-550, 1991.

[10] B. Pedersen, J. Chirravuri, and W. J. Miniscalco, "Gain and noise penalty for detuned 980-nm pumping of erbium-doped fiber power amplifiers," Photon. Tech. Lett., vol. 4, no. 4, pp. 351-353.

[11] O. Lumholt, K. Dybdal, C. C. Larsen, S. Dahl-Petersen, K. Sch 1 "usler, A. Bjarklev, J. H. Povlsen, T. P. Rasmussen, and K. Rottwitt, "ErLa doped fibre amplifier, pumped at $1.47 \mu \mathrm{m}, "$ Proc. 17 th ECOC '91, Paris, 1991, pp. 285-288.

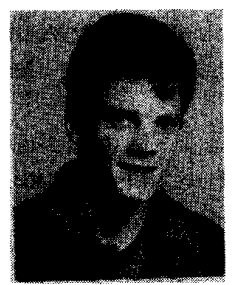

Ole Lumholt was born in Copenhagen, Denmark, on November 20,1963 . He received the M.Sc. degree in electrical engineering in 1990 from the Electromagnetics Institute, Technical University of Denmark.

In 1990, he joined the Electromagnetics Institute, Technical University of Denmark as an Associate Researcher. His research interests are in the field of active optical fibers and integrated optical waveguides.

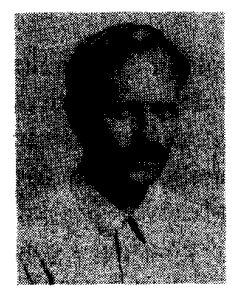

Jørn Hedegaard Povlsen was born on April 3, 1952. He received the M.Sc. degree in physics in 1982 from the H. C. $1 O$ rsted Institute of Copenhagen, Denmark.

In 1983 he joined the Electromagnetics Institute, Technical University of Denmark as an Associate Researcher. His research interests are in the field of characterization of active and passive optical waveguides and in the field of MQW structures.

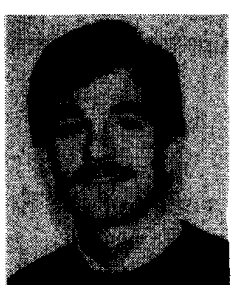

Kim Schüsler was born in Brlo ndby, Denmark, on May 14, 1962. He received the M.Sc. degree in electrical engineering in 1989 from the Industrial Acoustics Laboratory, Institute of Manufacturing Engineering, Technical University of Denmark.

In 1989 he joined the NKT Research Center A/S. His research interests are in the field of active optical fibers, optical networks, and optical sensors.

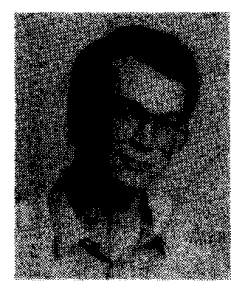

Anders Bjarklev was born in Roskilde, Denmark, on July 2, 1961. He received the M.Sc. degree in electrical engineering in 1985 from the Electromagnetics Institute, Technical University of Denmark. He received the Ph.D. degree from the Technical University of Denmark in 1988.

He became Associate Professor at the Electromagnetics Institute, Technical University of Denmark in 1992. His research interests are primarily within the field of characterization of active and passive optical waveguides.

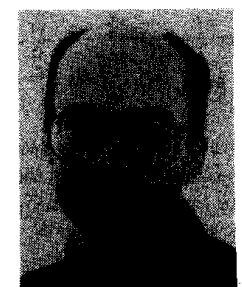

Svend Dahl-Petersen was born in Copenhagen, Denmark, on September 29, 1958. He received the M.Sc. degree in 1985 from University of Copenhagen with the major in physics and the minor in chemistry.

From 1985 to 1992 he was engaged at NKT Research Center working with $R \& D$ in the areas of high-temperature superconductors and fiberoptic communications. From 1989 to 1992 he was project manager for the coordinated Danish fiber amplifier project. In 1992 he was engaged by NKT Elektronik and he is now working as group leader of the TV section of the R\&D department.

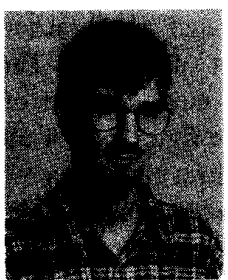

Thomas Rasmussen was born in Copenhagen, Denmark, on November 3, 1965. He received the M.Sc. degree in electrical engineering in 1991 from the Electromagnetics Institute, Technical University of Denmark. He is working on the Ph.D. degree at the Electromagnetics Institute, Technical University of Denmark, on integrated optical waveguides.

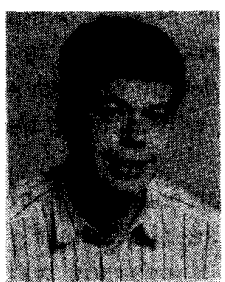

Karsten Rottwitt was born in Odense, Denmark, on November 13, 1965. He received the M.Sc degree in electrical engineering in 1990 from the Electromagnetics Institute, Technical University of Denmark. He is working on the Ph.D. degree at the Electromagnetics Institute, Technical University of Denmark, on soliton communication systems. 